\title{
Terapia dietetica del paziente con insufficienza renale cronica in terapia conservativa: più importanti della qualità sono la quantità e la frequenza del consumo
}

\author{
Franca Pasticci, Gianni Bellomo, Daniela Ricciardi, Antonio Selvi \\ UOC Nefrologia e Dialisi, USL Umbria 1, Pantalla di Todi (PG)
}

\begin{abstract}
Dietary management of chronic kidney disease
Diet in chronic kidney disease (CKD) is a component of medical therapy and in recent years the term diet has been replaced by nutritional-dietary therapy.

Food modulation permits to control metabolic changes that gradually will be established during the disease and to reduce complications that may arise delaying renal death and the need for replacement therapy. Protein restriction is the key point of the diet in conservative management of CKD. Many Authors state that diet could induce malnutrition, but literature shows that if the diet is well formulated and if it is followed by a properly trained staff, the patient's nutritional status is well preserved.

In our center nutritional-dietary therapy recommended in CKD stage 4-5 is a diet with 0.6-0.7 g of protein per $\mathrm{kg}$ of body weight, using low-protein food.

The approach we propose is a weekly chart with quantity and frequency of food agreed with the patient. The diet takes into account the national Guidelines for nutrition and the principles of the diet for the nutritional management of patients with CKD. Then, according to the habits, the tastes and the needs of the patients, the amounts of food and weekly frequencies are fixed. In this way, in our experience, we can meet the needs of our patients and have a better compliance.
\end{abstract}

Keywords: CKD, Dietary phosphorus, Dietary sodium, Low-protein diet

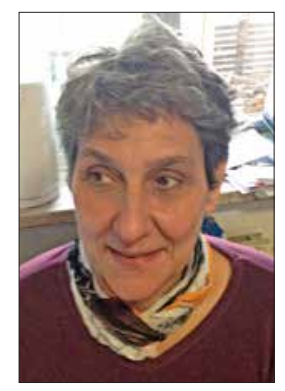

Franca Pasticci
La dieta, nella malattia renale cronica, è parte integrante della terapia medica e, negli ultimi anni, il termine dieta è stato sostituito da quello di Terapia dietetico-nutrizionale (1).

La modulazione degli alimenti permette di controllare le alterazioni metaboliche che si instaurano progressivamente nel corso della malattia e di contrastare le complicazioni che possono presentarsi, ritardando, così, la morte

Accepted: March 18, 2016

Published online: April 15, 2016

Indirizzo per la corrispondenza:

Dr.ssa Franca Pasticci

Unità Organica Complessa di Nefrologia e Dialisi - USL Umbria 1 Via del Buda

06059 Pantalla di Todi (PG)

franca.pasticci@uslumbria1.it renale (2) e la necessità della terapia sostitutiva (3). Nell'insufficienza renale cronica (IRC) in fase conservativa il cardine della dieta è la restrizione delle proteine (4). Molti Autori contestano che la dieta potrebbe produrre malnutrizione, $\mathrm{ma}$ in letteratura è stato dimostrato che, se la dieta è ben formulata e se viene seguita da personale adeguatamente formato, lo stato nutrizionale del paziente è ben conservato (5).

$\mathrm{Nel}$ nostro centro, la terapia dietetico nutrizionale consigliata nella maggior parte dei casi alle persone con IRC allo stadio 4-5 è quella classica, che prevede $0,6-0,7 \mathrm{~g}$ di proteine per kg di peso corporeo, con l'utilizzo di alimenti ipoproteici.

L'approccio che proponiamo è uno schema settimanale con quantità e frequenza degli alimenti concordata con il paziente. Si tengono in considerazione le Linee Guida nazionali per una corretta alimentazione (6) e i principi della dieta per la gestione nutrizionale del paziente con IRC (7). Poi, in base alle abitudini, ai gusti e alle necessità del paziente, vengono stabilite quantità degli alimenti e frequenze settimanali. In questo modo, secondo la nostra esperienza, riusciamo a soddisfare maggiormente le persone e a ottenere una buona adesione alle indicazioni. 


\section{Discussione}

La terapia dietetica nel corso della malattia renale prevede un corretto apporto di energia al fine di mantenere o di portare il peso corporeo in intervalli più vicini possibile alla normalità. Per definire la normalità, come per la popolazione generale, si fa riferimento all'indice di massa corporea, cioè al rapporto tra peso in $\mathrm{kg}$ e altezza in metri al quadrato. Un $\mathrm{BMI}$ tra 20 e $25 \mathrm{~kg} / \mathrm{m}^{2}$ viene definito normale, ma, in letteratura, molte fonti raccomandano che, nei pazienti con IRC, sia perseguito un BMI maggiore di $23 \mathrm{~kg} / \mathrm{m}^{2}$, in quanto meno associato al problema della malnutrizione calorico-proteica o Protein Energy Wasting (PEW) (8).

Le Linee Guida disponibili in letteratura suggeriscono che l'apporto energetico sia pari o superiore a 30-35 calorie per kg di peso corporeo.

Nella nostra esperienza, anche apporti energetici più contenuti sono in grado di mantenere positivo il bilancio energetico: probabilmente l'età avanzata e l'inattività fisica sono motivi importanti. Nella definizione dei fabbisogni, la storia dietetica è uno strumento fondamentale. La storia dietetica (9) è un'intervista strutturata, attraverso la quale si fa un inquadramento bio-psico-sociale del paziente e si quali-quantificano le sue abitudini alimentari, mettendole, poi, a confronto con i fabbisogni stimati dalle formule presenti in letteratura.

Nella pratica clinica, a seconda del risultato che si vuole ottenere (mantenimento, riduzione o aumento del peso), la storia dietetica permette di concordare con il paziente le variazioni necessarie. Occorre, però, ricordare che, nelle fasi avanzate della malattia renale, l'energia deve essere sufficiente per evitare il catabolismo a scopo energetico delle proteine introdotte. Per tale motivo, una dieta ipocalorica risulta poco indicata in queste fasi.

Accanto all'energia è fondamentale che sia valutato ed eventualmente modificato l'apporto di proteine. Le Linee Guida internazionali prevedono un apporto tra $0.8 \mathrm{~g}$ e $0.6 \mathrm{~g}$ per $\mathrm{kg}$ in relazione all'avanzamento della malattia e anche a quello che il paziente riesce a mettere in pratica (10). Degno di nota è che una restrizione anche se modesta rispetto alle abitudini attuali ( $0.2 \mathrm{~g} / \mathrm{kg}$ di proteine) è in grado di determinare effetti sul controllo dell'azotemia e delle altre alterazioni metaboliche (11). Per tale motivo, nella nostra pratica clinica, applichiamo una restrizione graduale dell'apporto proteico basata sulla storia dietetica.

Ancora una volta, è necessario ricordare che, in caso di restrizioni più marcate, deve essere data importanza all'apporto calorico perché le proteine siano utilizzate a scopo plastico o riparatore e non come fonti di energia.

In Italia, la disponibilità di alimenti ipoproteici (12) permette di organizzare la dieta rispettando le abitudini della dieta mediterranea, che si basa sui cereali (pasta e pane in particolare), oltre che su frutta, verdura e olio extravergine d'oliva. Negli ultimi anni, è cresciuto il numero delle aziende che producono alimenti ipoproteici e, oggi, è possibile trovare pasta, grissini, pane, fette biscottate, biscotti, snack e così via; inoltre, abbiamo la disponibilità di farina ipoproteica, con la quale si possono confezionare dolci o alimenti tipici, come tigelle o torte al testo. Infine, la palatabilità di questi prodotti è stata molto migliorata e se, una volta, il principale difetto della pasta ipoproteica era che si scuoceva rapidamente, oggi, grazie all'aggiunta di fibre e ad accorgimenti in fase di cottura, il problema è quasi risolto. L'aggiunta di fibre rende questa pasta adatta anche alle persone con diabete, in quanto rallenta l'assorbimento dell'amido, componente principale.

La disponibilità dei prodotti ipoproteici permette di lasciare porzioni di proteine animali più abbondanti, e, di conseguenza, di fornire aminoacidi di più elevato valore biologico, nonché di vitamine del gruppo $B$ e di sali minerali.

Infatti, gli alimenti di origine animale sono distinti in due gruppi:

- $\quad$ carne e derivati, pesce e derivati, uova: sono caratterizzati dal contenuto di ferro (eme);

- latte e derivati, che forniscono calcio (importante per la salute delle ossa).

Mediamente, $100 \mathrm{~g}$ di carne magra, che sia bianca o rossa, contengono $20 \mathrm{~g}$ di proteine; le carni grasse hanno un contenuto minore di proteine. È noto che i grassi contenuti nella carne rossa sono soprattutto saturi e aumentano la sintesi del colesterolo. Nella nostra pratica, consigliamo di consumare regolarmente carni magre, sia bianche che rosse, di consumare solo raramente carni grasse e di evitare le frattaglie e le interiora, in particolare se il colesterolo è elevato.

I derivati della carne sono salumi, affettati, carne in scatola e carne affumicata. Questi alimenti presentano due tipi di problemi: il primo è l'elevato contenuto di sodio, mentre il secondo problema è la possibilità che contengano additivi, in particolare a base di fosforo o potassio. In particolare, salumi e affettati sono molto palatabili e la maggior parte delle persone in Italia li consuma anche quotidianamente. Le persone con IRC si comportano come tutti e, anche quando interviene un'avversione per la carne, questi alimenti, in genere, sono ben accetti.

Come comportarsi per il sodio: si possono scegliere alimenti a minore contenuto. Per esempio, i salumi cotti come il prosciutto cotto o la mortadella hanno un contenuto di sodio pari a circa $1 / 3$ rispetto a quello del prosciutto crudo o del salame. Infatti, in questi ultimi, il sale ha il compito di conservante e deve essere in quantità tali da garantire la conservabilità. II prosciutto cotto o la mortadella, invece, sono alimenti deperibili in poco tempo. L'industria sta provando a produrre alimenti con ridotto contenuto di sodio, in particolare per le carni conservate in scatola. Occorre insegnare a scegliere e spesso è possibile soddisfare i gusti.

Per il pesce, si possono fare ragionamenti analoghi. II pesce propriamente detto è quello con le lische e va distinto da molluschi o crostacei. II pesce contiene una quantità di proteine in genere simile alla carne, $18-22 \%$; molluschi e crostacei ne 
contengono poco più della metà (12-15\%). Le Linee Guida per una corretta alimentazione raccomandano di consumare pesce 2-3 volte a settimana, perché contiene acidi grassi insaturi benefici. Molluschi e crostacei possono sostituire il pesce, ma è da considerare che contengono maggiori quantità di fosforo e colesterolo. Per il pesce conservato valgono le stesse indicazioni riportate per la carne (Tab. I).

L'alimento di riferimento per definire la qualità proteica è l'uovo. Questo alimento è spesso diffamato per il suo contenuto di colesterolo. In realtà, formaggi, salumi grassi o, ancora, prodotti da forno con grassi indefiniti possono essere più pericolosi. Nella pratica, l'uovo fornisce ottimi aminoacidi per la sintesi proteica e a basso costo. Se è necessario ridurre il colesterolo alimentare, può essere un suggerimento valido usare 2 bianchi e 1 rosso. Questo accorgimento può essere utile anche per ridurre il fosforo, visto che è contenuto esclusivamente nel rosso.

L'altro gruppo di alimenti importante per le proteine è costituito da latte e derivati, che, come detto, sono fonti di calcio (Tab. II).

È utile mettere in evidenza come, a mano a mano che il latte si asciuga e diventa ricotta, mozzarella o parmigiano, si riduce l'acqua e si concentrano proteine e grassi. Per questo motivo, si passa dal 3.5\% di proteine nel latte al 10\% nella ricotta, al $20 \%$ nella mozzarella e al $36 \%$ nel parmigiano. Latticini e formaggi hanno come ingrediente il sale: anche in questo caso, in generale, più sono stagionati più sale contengono.

Nelle Linee Guida per una corretta alimentazione italiana sono suggerite 3 porzioni di latte o yogurt al giorno e 3 porzioni a settimana di derivati del latte. Nell'IRC, questi alimenti devono essere limitati, perché forniscono un elevato apporto di fosforo. Per tale motivo, nel nostro centro, abbiamo ridotto la frequenza del latte/yogurt da 3 a 1 volta al giorno con la possibilità di sostituirli con 2 cucchiaini di formaggio grattugiato sui primi piatti; come secondo piatto, 2 volte a settimana lasciamo il consumo di formaggi, preferibilmente freschi.

Ultimi ma importanti alimenti fonti di proteine sono i legumi e la frutta secca, utilizzati regolarmente nelle diete vegetariane per raggiungere un apporto adeguato di proteine, ferro e calcio.

Questi alimenti sono spesso criticati per l'elevato conte-

TABELLA I - Carne, pesce e uova. Contenuto di nutrienti per porzione (19)

\begin{tabular}{|c|c|c|c|c|c|c|c|c|}
\hline & g & $\begin{array}{l}\text { Energia } \\
\text { calorie }\end{array}$ & Proteine $\mathrm{g}$ & Lipidi g & Calcio mg & Sodio $\mathrm{mg}$ & Potassio mg & Fosforo $\mathrm{mg}$ \\
\hline Vitellone magro & 100 & 113 & 21.3 & 3.1 & 11 & 89 & 360 & 175 \\
\hline Bistecca di maiale & 100 & 157 & 21.3 & 8 & 8 & 56 & 290 & 160 \\
\hline Uova & 100 & 128 & 12.4 & 8.7 & 48 & 137 & 133 & 210 \\
\hline Tonno sott'olio & 100 & 192 & 25.2 & 10.1 & 7 & 316 & 301 & 205 \\
\hline Prosciutto cotto & 100 & 215 & 20 & 15 & 6 & 648 & 227 & 250 \\
\hline Prosciutto di Parma & 100 & 268 & 25.5 & 18.4 & 16 & 2578 & 373 & 261 \\
\hline
\end{tabular}

TABELLA II - Latte e derivati. Contenuto di nutrienti per porzione (19). La porzione è definita in base ai prodotti presenti in commercio. Per esempio, $60 \mathrm{~g}$ di mozzarella sono la metà di una mozzarella in busta e $100 \mathrm{~g}$ di ricotta sono la porzione comunemente in vendita

\begin{tabular}{|c|c|c|c|c|c|c|c|c|}
\hline & g & $\begin{array}{l}\text { Energia } \\
\text { calorie }\end{array}$ & $\begin{array}{l}\text { Proteine } \\
\text { g }\end{array}$ & Lipidi g & Calcio mg & Sodio $\mathrm{mg}$ & Potassio mg & $\begin{array}{c}\text { Fosforo } \\
\text { mg }\end{array}$ \\
\hline Latte parzialmente scremato & 125 & 58 & 4 & 2 & 150 & 53 & 213 & 118 \\
\hline Latte intero & 125 & 79 & 4 & 4.5 & 150 & 59 & 188 & 119 \\
\hline Yogurt intero alla frutta & 125 & 110 & 3.5 & 4.2 & 139 & 60 & 188 & 109 \\
\hline Fontina & 50 & 172 & 12.3 & 13.5 & 435 & 343 & 45 & 280 \\
\hline Mozzarella & 60 & 152 & 11.2 & 11.7 & 96 & 120 & 87 & 210 \\
\hline Parmigiano & 10 & 39 & 3.4 & 2.8 & 116 & 56 & 10 & 68 \\
\hline Ricotta di mucca & 100 & 146 & 9 & 11 & 295 & 78 & 119 & 237 \\
\hline
\end{tabular}


TABELLA III - Dieta per un paziente in terapia dietetica conservativa. Soggetto di $60 \mathrm{~kg}$. Medie settimanali: calorie 1782 , proteine $38 \mathrm{~g}$ (8.6\%), di cui $26 \mathrm{~g}$ animali con rapporto fosforo-proteine 12, lipidi totali $33 \%$, lipidi saturi $6 \%$ dell'energia, colesterolo $132 \mathrm{mg} /$ die, glucidi 59.4\%, fosforo $622 \mathrm{mg}$, potassio $1867 \mathrm{mg}$, sodio degli alimenti $680 \mathrm{mg}$, fibra $21 \mathrm{~g}$

\begin{tabular}{llcc}
\hline & Alimento & Porzione & Frequenza \\
\hline Latte & $\begin{array}{l}\text { Latte parzialmente scremato, yogurt intero o } \\
\text { alla frutta oppure 2 cucchiaini di parmigiano }\end{array}$ & $125 \mathrm{~g}$ & 1 al giorno
\end{tabular}

Derivati del latte

Carne, pesce, uova

Pasta

Cereali e derivati

Ortaggi

Frutta

Condimenti

Burro, margarina

Carne fresca
Pesce fresco
Affettati
Uova

Freschi

Secchi

Pane ipoproteico

Insalate

Ortaggi

$\begin{array}{ll}50 \mathrm{~g} & 2 \text { porzioni a settimana } \\ 30 \mathrm{~g} & \end{array}$

Formaggi stagionati

Pasta, riso, farina di mais

Pasta ipoproteica asciutta

Ciambelle, crostate ipoproteiche

Biscotti secchi, fette biscottate ipoproteiche

Fresca di stagione

1 cucchiaio da $10 \mathrm{~g} 4$ porzioni al giorno
$150 \mathrm{~g}$

2 porzioni al giorno

3 porzioni a settimana

2 porzioni a settimana

1 porzione a settimana

2 a settimana

2 porzioni a settimana

2 porzioni a settimana

5 porzioni a settimana

4 porzioni al giorno

2 porzioni al giorno

$\begin{array}{ll}150 \mathrm{~g} & 2 \text { porzioni a settimana } \\ 40 \mathrm{~g} & \\ 50 \mathrm{~g} & 2 \text { porzioni a settimana } \\ 80 \mathrm{~g} & 5 \text { porzioni a settimana } \\ 50 \mathrm{~g} & 4 \text { porzioni al giorno } \\ 50 \mathrm{~g} & \\ 40 \mathrm{~g} & \\ 100 \mathrm{~g} & 2 \text { porzioni al giorno } \\ 150 \mathrm{~g} & \end{array}$

$150 \mathrm{~g}$

(

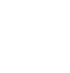

(1)

Zucchero

Zucchero

Marmellata, miele
$20 \mathrm{~g}$

$25 \mathrm{~g}$ 
è assorbito al massimo al 60\% (meno nel caso di alimenti di origine vegetali per la presenza di fitati). Un esempio è il lievito chimico usato nella lievitazione dei dolci: nella preparazione casalinga, una dose per mezzo kg di farina apporta una quantità di fosforo pari a $1589 \mathrm{mg}$. Se le persone consumano abitualmente biscotti o dolci, potrebbero introdurre molto fosforo.

Altre fonti sono i polifosfati aggiunti nella preparazione di surimi, prosciutto cotto, formaggini, sottilette e così via. Questi additivi sono usati sia per trattenere acqua (di conseguenza i salumi non perdono peso nella stagionatura) che per fondere i formaggi (che restano morbidi e facilmente spalmabili). Ancora, possiamo trovare fosforo nella panatura di pesce, formaggi e carne. È importante insegnare al paziente a leggere le etichette degli alimenti e a riconoscere gli additivi da evitare.

Per quanto riguarda il potassio, la restrizione dell'apporto non è sempre necessaria. Particolare attenzione va posta nel caso che siano prescritti farmaci come ACE o sartanici o diuretici risparmiatori di potassio. Va combattuta la stitichezza, in quanto il potassio viene eliminato anche per via fecale. Infine, l'acidosi può essere causa di un aumento dei valori del potassio nel sangue. Quando è necessario, occorre fare attenzione alla quantità di frutta e verdura che si consuma, evitare o ridurre la frutta e la verdura più ricca di potassio e mettere in atto alcuni accorgimenti che consentono di ridurre il potassio negli alimenti. In particolare, durante la cottura in acqua il potassio si scioglie nell'acqua: buttando l'acqua di cottura si elimina parte del potassio; più acqua si usa e più potassio si elimina.

L'apporto di sodio nella malattia renale deve essere pari a 2-2.5 g al giorno, che corrispondono a quanto raccomandato per la popolazione sana. La riduzione del sodio è importante per contrastare l'ipertensione e il sovraccarico idro-salino, per migliorare la risposta ai farmaci anti-proteinurici e nelle malattie cardiovascolari (16). Secondo l'Istituto Nazionale per I'Alimentazione, circa il $60 \%$ del sale che introduciamo deriva da prodotti da forno (come pane, cracker, ma anche dolci o biscotti), da alimenti conservati (affettati, formaggi e alimenti in scatola) e dai cibi pronti (dagli alimenti cotti agli alimenti surgelati già pronti), che la maggior parte della popolazione sta imparando a consumare quotidianamente. Chiedere ai pazienti di preparare il cibo in casa con alimenti freschi o surgelati, riducendo il sale aggiunto ma aggiungendo erbe e spezie e aromi per migliorare il sapore, è una sfida.

A questo punto, possono essere permesse piccole porzioni di alimenti "salati". Se, infatti, $100 \mathrm{~g}$ di crudo contengono $2500 \mathrm{mg}$ di sodio, $50 \mathrm{~g}$ ne contengono $1250 \mathrm{mg}$. Considerando le porzioni comunemente consumate, i $50 \mathrm{~g}$ proposti sono poca cosa. Diventa importante insegnare come rendere questa porzione accettabile; per esempio, non dovrebbe mai costituire il pasto, ma seguire un primo piatto, oppure potrebbe essere utilizzata come ingrediente; infine, deve sempre essere abbinata a pane, anche ipoproteico, e verdure.

Nel corso degli anni, abbiamo messo a punto una proposta di dieta a schema settimanale (Tab. III), che permette, a nostro parere, di gestire meglio i gusti e le necessità delle persone seguite presso il nostro centro. In uno schema del genere nulla è vietato. II nostro motto è: frequenza e quantità sono più importanti della qualità. Questo ci aiuta a stringere una complicità con le persone e ad avere una buona adesione al trattamento (Tabb. IV, V).

\section{Follow up}

Dato che la malnutrizione è il problema che più spesso viene imputato alle diete ipoproteiche, la valutazione seriale dello stato di nutrizione diventa fondamentale.

Tra gli indici antropometrici, il peso è quello che valuta l'energia: a seconda del risultato che vogliamo avere, riduzione o incremento del peso, dovremo modificare o confermare gli apporti energetici. Accanto a questo vengono rilevate le circonferenze della vita e brachiale e le pliche tricipitale e sottoscapolare (17) per la valutazione seriale.

La valutazione dietetica viene fatta ad ogni incontro attraverso la replica della storia dietetica oppure la ripetizione ad ogni incontro del 24 ore recall (metodo veloce per stimare gli apporti dietetici nelle 24 ore precedenti). II primo incontro è

TABELLA IV - La composizione bromatologica della dieta è stata calcolata sulla seguente distribuzione settimanale dei secondi piatti, che può essere usata come esempio

\begin{tabular}{llclc}
\hline & Pranzo & g & Cena & g \\
\hline Lunedi & Merluzzo & 100 & Uovo & n. 1 \\
Martedì & Ceci secchi & 40 & Mozzarella & 50 \\
Mercoledì & Tacchino & 100 & Prosciutto cotto & 50 \\
Giovedì & Maiale & 100 & Mortadella & 40 \\
Venerdì & Palombo & 100 & Stracchino & 50 \\
Sabato & Fagioli secchi & 40 & Uovo & n. 1 \\
Domenica & Vitello & 50 & Pollo & 100 \\
\hline
\end{tabular}

TABELLA V - Esempio di dieta

\begin{tabular}{lc}
\hline Quantità giornaliera & g \\
\hline Latte parzialmente scremato & 125 \\
Pane ipoproteico fresco & $50+50+50+50$ \\
Pasta ipoproteica & 80 \\
Carne magra & $100+50$ \\
Olio extravergine d'oliva & 40 \\
Verdure (pomodori, bieta, lattuga) & 300 \\
Frutta (mela, arancia) & 300 \\
Marmellata & 25 \\
Zucchero & 20
\end{tabular}


particolarmente importante e richiede molto tempo, in quanto occorre inquadrare il paziente sia dal punto di vista delle abitudini dietetiche che sul versante bio-psico-sociale.

Fondamentali sono anche gli indici biochimici e i parametri urinari (18). In particolare, la raccolta delle urine nelle 24 ore, oltre a definire la clearance della creatinina, permette di verificare l'adesione alla dieta: dall'azoturia e dalla sodiuria è possibile calcolare immediatamente gli apporti di proteine e sale.

\section{Disclosures}

Financial support: No financial support was received for this submission. Conflict of interest: The authors have no conflict of interest.

\section{Bibliografia}

1. Terapia nutrizionale nell'IRC in fase conservativa: suggerimenti di pratica clinica e di applicazione delle Linee Guida, Best Practice. Società Italiana di Nefrologia 2012, 7 Settembre 2012.

2. Identificazione, prevenzione e gestione della malattia renale cronica nell'adulto. Linea Guida 23 ISS 2012 (http://www.snlgiss.it/cms/files/LGrenale_finaleL.pdf. Accessed April 9, 2016).

3. Fouque D, Laville M. Low protein diets for chronic kidney disease in non diabetic adults. Cochrane database Syst Rev 2009;(3):CD001892.

4. Klahr S, Levey AS, Beck GJ, et al. The effects of dietary protein restriction and blood-pressure control on the progression of chronic renal disease. Modification of Diet in Renal Disease Study Group. N Engl J Med 1994;330:877-84.

5. Levey AS, Greene T, Sarnak MJ, et al. Effect of dietary protein restriction on the progression of kidney disease: long-term follow-up of the Modification of Diet in Renal Disease (MDRD) Study. Am J Kidney Dis 2006;48(6):879-88.

6. Linee Guida per una sana alimentazione italiana, a cura del Ministero Politiche Agricole e Forestali e INRAN. Revisione 2003 (http://www. salute.gov.it/imgs/C_17_pubblicazioni_652_allegato.pdf).
7. Linee Guida per la terapia conservativa dell'insufficienza renale cronica. G Ital Nefrol 2003;(Suppl. 24):S48-60.

8. Fouque D, et al. EBPG Guideline on Nutrition Nephrology Dialysis Transplantation. Volume 22(Suppl. 2):ii45-87.

9. Ruolo del dietista nella gestione nutrizionale del paziente con malattia renale cronica. Gruppo di lavoro ANDID Malattie Renali (http://www.andid.it/andid/posizioni-dell-andid/item/ ruolo-del-dietista-nella-gestione-nutrizionale-del-pazientecon-malattia-renale-cronica).

10. Kopple JD. National kidney foundation K/DOQI clinical practice guidelines for nutrition in chronic renal failure. Am J Kidney Dis 2001; 37(1 Suppl 2):S66-70

11. Mitch WE, Remuzzi G. Diets for patients with chronic kidney disease, still worth prescribing. J Am Soc Nephrol 2004;15(1):234-7.

12. D'Alessandro C, Rossi A, Innocenti M, et al. Dietary protein restriction for renal patients: don't forget protein-free foods. J Ren Nutr 2013;23(5):367-71.

13. Cupisti A, Morelli E, Meola M, Barsotti M, Barsotti G. Vegetarian diet alternated with conventional low-protein diet for patients with chronic renal failure. J Renal Nutr 2002:12:32-7.

14. Cupisti A, et al. Phosphate control in chronic uremia: Don't forget diet. J Nephrol 2003;16(1):29-33.

15. Cupisti A, Kalantar-Zadeh K. Management of Natural and Added Dietary Phosphorus Burden in Kidney Disease. Semin Nephrol 2013:33:180-90.

16. Locatelli F, Del Vecchio L. Protein restriction: a revised old strategy with new opportunities? Nephrol Dial Transplant 2014;29(9):1624-7.

17. Pasticci F, Fantuzzi AL, Pegoraro M, et al. Nutritional management of stage 5 chronic kidney disease. J Ren Care 2012; 38(1):50-8.

18. Bellizzi V, Bedogni G, Quintaliani G. Compliance alla dieta nell'insufficienza renale cronica. G Ital Nefrol 2008;25(Suppl. 42):S45-9.

19. Banca Dati di Composizione degli Alimenti per Studi Epidemiologici in Italia (BDA) 2015. 